\title{
On the partial wave amplitude of Coulomb scattering in three dimensions*
}

\author{
Qiong-gui $\operatorname{Lin}^{\dagger}$ \\ Department of Physics, Zhongshan University, Guangzhou 510275, \\ People's Republic of China $a^{\ddagger}$ \\ and \\ China Center of Advanced Science and Technology (World Laboratory), \\ P.O.Box 8730, Beijing 100080, People's Republic of China
}

The partial wave series for the Coulomb scattering amplitude in three dimensions is evaluated in a very simple way to give the closed result.

PACS number(s): 03.65.Nk

*published in Am. J. Phys. 68 (2000) 1056-1057.

†E-mail: qg_lin@163.net, stdp@zsu.edu.cn

${ }^{\ddagger}$ Mailing address 
The scattering of particles by a Coulomb field is an important subject in quantum mechanics as well as in classical mechanics, because the Coulomb field describes real physical interations between charged particles, say, electrons and nucleuses. In the nonrelativistic quantum mechanics, the problem is described by a Schrödinger equation. In ordinary three-dimensional space, this equation can be solved in rotational parabolic coordinates, and the scattering amplitude is obtained in a closed form. As a special case of a centrally symmetric field, the Schrödinger equation can also be solved in the spherical coordinates, and the scattering amplitude is obtained as a partial wave series. This has been well known for a long time, and the solutions can be found in the popular textbooks of quantum mechanics [1,2]. However, it appears that the partial wave amplitude is not evaluated in the textbooks. Were it not for the solution in the parabolic coordinates, how can one directly sum up the partial wave series to obtain the closed form? A solution can be found in an early paper of Mott [3]. However, the calculation is not well known since it is not given even in the famous monograph on collision theory by that author and Massey [4]. Other books on scattering theory do not deal with the matter either $[5,6]$. The purpose of this note is to evaluate the partial wave series in a simpler way. The method is easy for the students and thus may be of some interest for the pedagogical purpose.

Let us write the Hamiltonian in the form

$$
H=\frac{\mathbf{p}^{2}}{2 \mu}-\frac{\kappa}{r}
$$

where $\mu$ is the reduced mass of the system, and $\kappa>0(\kappa<0)$ corresponds to an attractive (repulsive) force. The partial wave scattering amplitude obtained by solving the problem in spherical coordinates reads $[1,2]$

$$
f(\theta)=\frac{1}{2 i k} \sum_{l=0}^{\infty}(2 l+1)\left(S_{l}-1\right) P_{l}(\cos \theta),
$$

where $k=\sqrt{2 \mu E} / \hbar(E>0$ is the energy of the incident particle), and

$$
S_{l}=\exp \left(2 i \delta_{l}\right)=\frac{\Gamma(l+1-i \beta)}{\Gamma(l+1+i \beta)},
$$


where $\delta_{l}$ is the phase shift of the $l$ th partial wave, and $\beta=\mu \kappa / \hbar^{2} k=\kappa / \hbar v$ where $v$ is the incident velocity. Since [1,2]

$$
\sum_{l=0}^{\infty}(2 l+1) P_{l}(\cos \theta)=4 \delta(\cos \theta-1)
$$

we have for $\theta \neq 0$

$$
f(\theta)=\frac{1}{2 i k} \sum_{l=0}^{\infty}(2 l+1) S_{l} P_{l}(\cos \theta)
$$

We define

$$
g(x)=\sum_{l=0}^{\infty}(2 l+1) S_{l} P_{l}(x)
$$

and are going to evaluate it. Using the relation

$$
(2 l+1) P_{l}(x)=P_{l+1}^{\prime}(x)-P_{l-1}^{\prime}(x),
$$

where $P_{-1}(x) \equiv 0$, and define an auxiliary function

$$
G(x)=\sum_{l=0}^{\infty} S_{l}\left[P_{l+1}(x)-P_{l-1}(x)\right]
$$

we have

$$
g(x)=G^{\prime}(x)
$$

Next using the ralation

$$
(2 l+1) x P_{l}(x)=(l+1) P_{l+1}(x)+l P_{l-1}(x),
$$

and the definition (8), we have

$$
x g(x)=\sum_{l=0}^{\infty} S_{l}\left[(l+1-i \beta) P_{l+1}(x)+(l+i \beta) P_{l-1}(x)\right]+i \beta G(x) .
$$

Then making use of the relation

$$
\Gamma(z+1)=z \Gamma(z)
$$


and Eq. (3), we have

$$
(l+1-i \beta) S_{l}=(l+1+i \beta) S_{l+1}, \quad(l+i \beta) S_{l}=(l-i \beta) S_{l-1} .
$$

Substituting these relations into Eq. (11), after some simple algebras we have

$$
x g(x)=g(x)+i \beta G(x)-i \beta S_{0}
$$

Taking Eq. (9) into account, we obtain

$$
(1-x) G^{\prime}(x)+i \beta G(x)=i \beta S_{0} .
$$

This is an ordinary differential equation for the auxiliary function $G(x)$. It can be easily solved to give

$$
G(x)=A \exp [i \beta \ln (1-x)]+S_{0}
$$

where $A$ is a constant of integration. To determine $A$ we consider the series (8) at $x=-1$. Note that $P_{l}(-1)=(-1)^{l}$ and $P_{-1}(x) \equiv 0$, only the term with $l=0$ contributes and so $G(-1)=-S_{0}$. Substituting this into the above result one can find $A$ and arrives at

$$
G(x)=-2 S_{0} \exp [i \beta \ln ((1-x) / 2)]+S_{0}
$$

It should be remarked that in getting Eq. (9) we have differentiated $G(x)$ term by term. This is not allowed at $x=1$ since $g(x)$ is obviously singular at the point. Thus Eq. (15) does not hold at $x=1$, and it is not surprising to find that the result (17) does not agree with the definition (8) at that point. By using Eq. (9) we can easily find $g(x)$. Setting $x=\cos \theta$ and multiplying it by the coefficient $1 / 2 i k$ we arrive at

$$
f(\theta)=\frac{\Gamma(1-i \beta)}{i \Gamma(i \beta)} \frac{\exp \left[i \beta \ln \sin ^{2}(\theta / 2)\right]}{2 k \sin ^{2}(\theta / 2)}
$$

This is the closed result obtained in the parabolic coordinates $[1,2]$. Two remarks about the result. First, we have dropped a $\delta(\cos \theta-1)$ term for $\theta \neq 0$ in the above calculation. Here the 
final result is more singular than $\delta(\cos \theta-1)$ when $\theta \rightarrow 0$, thus that term can indeed be dropped everywhere. Second, it has been argued that $f(\theta)$ in the form of Eq. (2) or (5) is divergent even when $\theta \neq 0[7]$. The final result (18) is well defined everywhere except at $\theta=0$, however. Therefore the above calculation may also be regarded as a regularization procedure. (The main point lies in the term by term differentiation pointed out above, which is mathematically not allowed even when $x \neq 1$.) The validity of this procedure is obvious because the final result (18) coincides with the one obtained from exact solutions in the parabolic coordinates. It should be pointed out that the calculation of Mott [3], though rather different from ours, involves some similar story.

Mathematically one can consider the Coulomb scattering problem in a general space dimension $d \geq 2$. In $d=2$ the problem has been solved in both parabolic coordinates [8-9] and polar coordinates [10]. The scattering amplitude is obtained in a closed form in the former case and as a partial wave series in the latter case. The method for summing up the partial wave series in $d=2$ is rather different from that in $d=3[10,11]$. In more general dimensions $d>3$ the problem has been solved in parabolic coordinates and a closed form for the scattering amplitude was obtained [12]. If one solves the problem in $d$-dimensional spherical coordinates, one would obtain for the scattering amplitude a partial wave series similar to Eq. (2), where the Legendre polynomials are replaced by the Gegenbauer ones. We expect that the method given by Mott or the one given here may be efficient for summing up that series as well.

The author is grateful to Professor W. Dittrich and Professor B. R. Holstein for communications and encouragement. This work was supported by the National Natural Science Foundation of China. 
[1] L. I. Schiff, Quantum Mechanics, 3rd ed. (McGraw-Hill, New York, 1968).

[2] L. D. Landau and E. M. Lifshitz, Quantum Mechanics, 3rd ed. (Pergamon, Oxford, 1977).

[3] N. F. Mott, "The polarization of electrons by double scattering," Proc. Roy. Soc. (London) A 135, 429-458 (1932).

[4] N. F. Mott and H. S. W. Massey, The Theory of Atomic Collisions, 3rd ed. (Oxford University, Oxford, 1965).

[5] R. G. Newton, Scattering Theory of Waves and Particles, 2nd ed. (Springer-Verlag, New York, 1982).

[6] M. L. Goldberger and K. M. Watson, Collisions Theory (Wiley, New York, 1964).

[7] L. Marquez, "On the divergence of the Rutherford scattering amplitude in terms of Coulomb phase shifts," Am. J. Phys. 40, 1420-1427 (1972).

[8] G. Barton, "Rutherford scattering in two dimensions," Am. J. Phys. 51, 420-422 (1983); Q.-G. Lin, "Scattering by a Coulomb field in two dimensions," ibid. 65, 1007-1009 (1997); M. J. Moritz and H. Friedrich, "Scattering by a Coulomb field in two dimensions," ibid. 66, 274 (1998).

[9] Q.-G. Lin, "Quantum-mechanical model for particles carrying electric charge and magnetic flux in two dimensions," Phys. Rev. A 59, 3228-3235 (1999).

[10] J. Law, M. K. Srivastava, R. K. Bhaduri, and A. Khare, "Mott scattering of anyons: some exact results," J. Phys. A 25, L183-L187 (1992).

[11] Q.-G. Lin, "Scattering of relativistic particles by a Coulomb field in two dimensions," Phys. Lett. A 260, 17-23 (1999).

[12] D. Yafaev, "On the classical and quantum Coulomb scattering," J. Phys. A 30, 6981-6992 (1997). 